\title{
A Case of Interstitial Pneumonia Antedating Rheumatoid Arthritis
}

\author{
SHINYA SUMIDA, AKIRA NAGATA, KAZUTOYO KANEKO, \\ MIKIO KUBOSHIRO, NAOTO KAMATANI, \\ TSUNEAKI SHIRAISHI, MAKO KATSUKI, \\ TOSHIHIKO HARADA, NAOTO TOKUNAGA* \\ AND MASARO KAJI* \\ Chikugo Municipal Hospital, Chikugo, 833 and \\ *Department of Internal Medicine, Kurume University School of Medicine, \\ Kurume, 830 Japan
}

Received for publication May 24, 1989

\begin{abstract}
Summary: We describe a case report of an interstitial pneumonia in which pulmonary involvement of the both lower lobes mimicked idiopathic interstitial pneumonia (IP) or bronchiolitis obliterans organizing pneumonia (BOOP). Its appearance antedated clinically apparent rheumatoid arthritis by one month and a half.
\end{abstract}

Key words: interstitial pneumonia - pulmonary involvement of RA-rheumatoid arthritis - cellular interstitial infiltrate - predonisolone therapy

\section{Introduction}

Extra-articular manifestations occur frequently in rheumatoid arthritis. Five different patterns of lung disease have been described: 1) diffuse interstitial fibrosis; 2) necrobiotic nodules; 3) pleuritis and pleural effusions; 4) obstructive airway disease including bronchiolitis obliterans; 5) pulmonary hypertention secondary to pulmonary arteritis (Turner et al. 1977). The diagnosis of rheumatoid lung involvement associated with clinically apparent rheumatoid arthritis is usually easy. However, we describe in this report the unusual occurrence of interstitial pneumonia prior to the onset of clinical rheumatoid manifestations. This interstitial pneumonia mimicked IIP or BOOP, and was detected in its early stages.

\section{Case Report}

A 43-year-old woman complained of productive cough, fever and exertional dyspnea since October, 1987. At physical examination on November18, marked fine crackles were heard on auscultation localized to the bilateral bases of the lungs but particularly prominent in the right lung. A chest roentgenogram revealed reticulonodular shadows in both lower lobes. CT scan demonstrated relatively high density areas in both lower lobes spreading from the pleura with a pneumo-bronchogram present. Transbronchial lung biopsy (TBLB) was then performed and was interpreted as showing interstitial pneumonia (Fig. 1). Routine and special stains of the sputum revealed no pathogens and cultures were negative. The skin reaction 


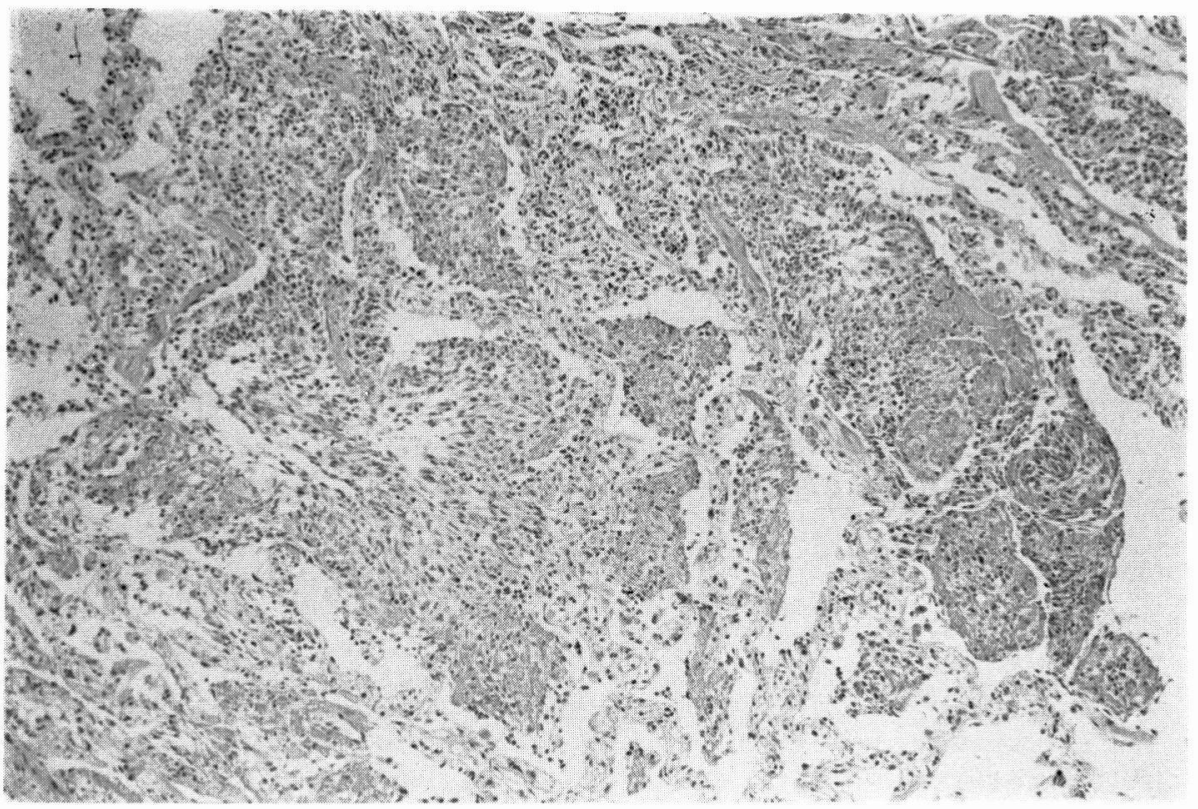

Fig. 1. Low power photomicrogragh of lung mass. Note alveolar exudate and mononuclear infiltration. $(\mathrm{H} \cdot \mathrm{E} \times 50)$

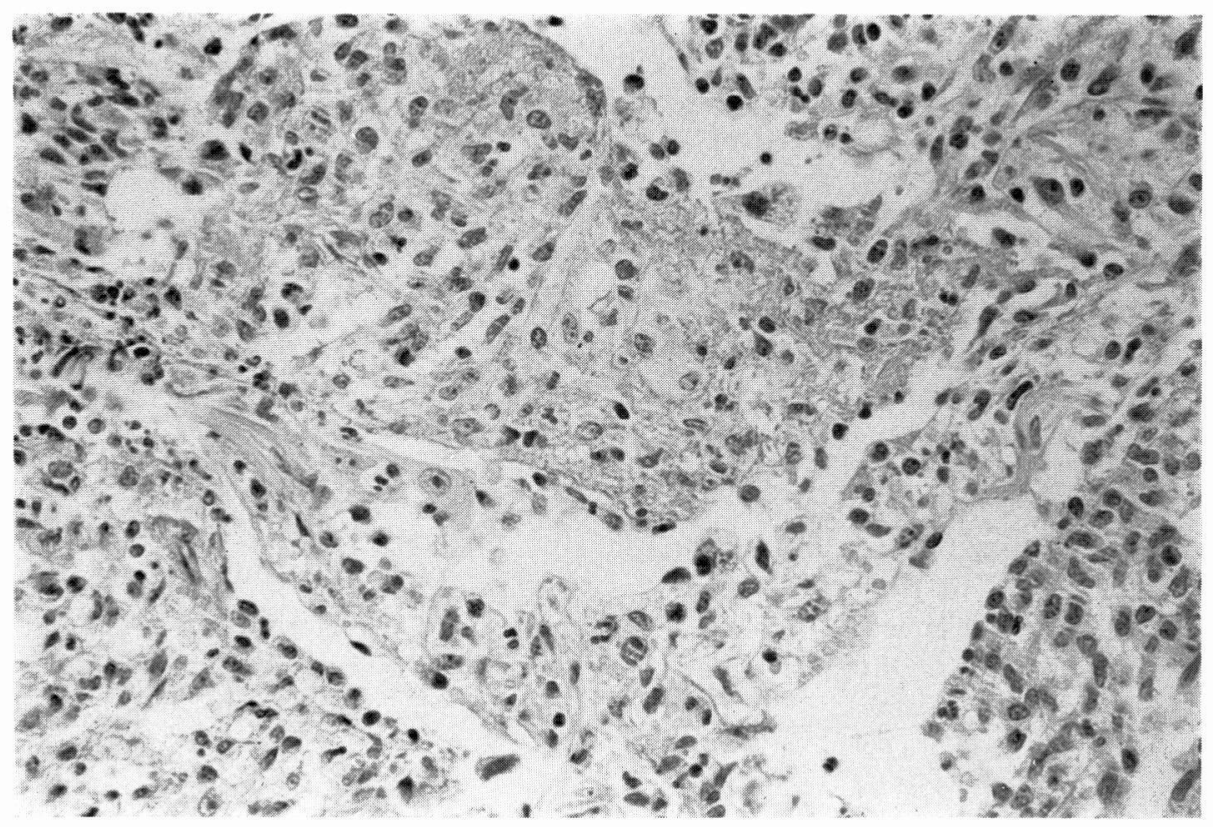

Fig. 2. High power view of mass. Note interstitial edema and septal thickening. $(\mathrm{H} \cdot \mathrm{E} \times 100)$ 
to purified protein derivative was negative.

On November 21, the patient experienced the onset of arthralgia of the right hip joint. Morning stiffness, polyarthralgia and painful swelling of both her hands as well as of the feet followed. The patient exhibited erythrocyte sedimentation rate of $142 \mathrm{~mm} /$ hour, her hematocrit was 30 , her hemoglobin $9.4 \mathrm{mg} / \mathrm{dl}$, and the rheumatoid factor titer was 1 in 117 . LE cells were not present and the antinuclear factor titer was less than 5 . Urinalysis was unremarkable. Radiographs of the hands did not show joint space narrowing or erosions. Lung function tests were normal. The diagnosis of interstitial pneumonia associated with rheumatoid arthritis was made. The patient was started on predonisolone sodium succinate $(40 \mathrm{mg}$ / day) and made a marked improvement.

\section{Discussion}

There are three lines of evidence leading to the conclusion that Usual Interstitial Pneumonia (UIP) in the patient preceded the manifestation of rheumatoid arthritis.

First and importantly, the histological appearence supported the diagnosis of UIP. The characteristic features of UIP, which were present, include alveolar exudate, septal thickening with hyaline membranes, and infiltrates of mononuclear cells (Fig. 2). Histological changes which occur in rheumatoid lung disease may be classified as: 1) pulmonary rheumatoid nodules, 2) usual interstitial pneumonia (UIP), 3) BOOP, 4) lymphoid hyperplasia and 5) cellular interstitial infiltrate (Yousem et al. 1985). The histology of this case revealed UIP; the cellular interstitial infiltrate indicated the early stage of UIP. Lung disease is commonly associated with rheumatoid arthritis, but lung disease antedating rheumatoid arthritis is rare. Reports of lung involvement at autopsy have varied from none in nine cases to eight in forty one cases (Walker at al. 1968; Kinjo, 1982).

Secondly, the patient developed polyarthritis after UIP. The manifestations of this case were compatible with the criteria for the diagnosis of rheumatoid arthritis of the American Rheumatology Association, as the patient described here satisfied their criteria as follows: 1) She presented morning stiffness with which continued for an hour at least; 2) She had joint swelling of more than three joints; 3) the wrist, MCP and PIP joints were involved.; 4) There was symmetrical joint involvement; and 5) presence of rheumatoid factor was established.

Thirdly, there was a good response to predonisolone therapy. As well known predonisolone has a remarkable efficacy in UIP especially in the early stages of UIP, as in this case. Immunological mecha nisms probably play very important roles in the response of UIP to predonisolone, as $\mathrm{RF}$ immunocomplexes can promote rheumatoid lung disease via activation of the classical pathway of complement (Hakala, 1986). Predonisolone could suppress this pathway and also decreases inflammation. Altogether, the evidence adduced that this case developed UIP one and a half months before the onset of rheumatoid arthritis.

\section{References}

Hakala, M. (1986). Diffuse interstitial lung disease in rheumatoid arthritis. Scand. J. Rheumatol. 15, 368-376.

Kinjo, M. (1982). Pulmonary manifestations of Rheumatoid Arthritis. Jpn. J. Chest. Dis. 20, 76-83.

Turner-Warwick, M. and Evance, R. C. (1977). Pulmonary manifestations of rheumatoid dis ease. Clin. Rheum. Dis. 3, 549-645.

Walker, W. C. and Wright, V. (1968). Pulmorary lesions and rheumatoid arthritis. Medicine 47, 501-520.

Yousem, S. A. and Colby, T. V. (1985). Lung biopsy in rheumatoid arthritis. Am. Rev. Respir. Dis. 131, 770-777. 